\title{
Gap solitons and soliton trains in finite-sized two-dimensional periodic and quasiperiodic photonic crystals
}

\author{
Ping Xie, Zhao-Qing Zhang, and Xiangdong Zhang \\ Department of Physics and Institute of Nano Science and Technology (INST), The Hong Kong University of Science and Technology, \\ Clear Water Bay, Hong Kong, China \\ (Received 16 September 2002; revised manuscript received 19 November 2002; published 13 February 2003)
}

\begin{abstract}
We demonstrate the existence of the gap solitons and soliton trains in finite-sized two-dimensional periodic nonlinear photonic crystals by using the mutiple-scattering approach with an iterative scheme. In 12-fold symmetric nonlinear quasicrystals, we also demonstrated the existence of symmetric, regular gap solitons, asymmetric single-soliton states, and two-solitons states. We revealed that the existence of symmetric, regular gap solitons in a 12-fold quasicrystal is limited by the geometrical size of the hexagon that forms the core of the dodecahedral cell, which is the building block of the quasicrystal.
\end{abstract}

DOI: 10.1103/PhysRevE.67.026607

PACS number(s): 42.70.Qs, 42.65.Tg, 63.20.Pw

In past years there has been a great deal of interest in photonic crystals made of linear materials [1]. They make the control and manipulation of light propagation possible and, therefore, can have vast implications in both quantum optics and optical devices. In order to employ the high-technology potential of photonic crystals, it is also important to achieve a dynamical tunability of their properties. This can be done by replacing the linear materials with nonlinear ones and by changing the light intensity in the photonic crystals [2]. An important phenomenon in the nonlinear photonic crystals is the existence of the nonlinearity induced localized states with frequencies in the forbidden gaps, which are usually called gap solitons. The term gap soliton was first introduced by Chen and Mills in their numerical study of onedimensional (1D) periodic superlattices [3]. Later, the subject of the gap solitons in two- and three-dimensional nonlinear photonic crystals has been studied by John and Akozbek [4] by using the coupled-mode theory, which is valid in small dielectric modulations. Recently, by using the numerical Green's function, Mingaleev and Kivshar [5] demonstrated the existence of stable single-gap solitons in 2D photonic crystals with large dielectric contrast ratio. A question remains open, whether stable multiple-soliton states exist in 2D periodic crystals, which exhibit multistability, in analogy to $1 \mathrm{D}$ periodic superlattices [6]. Parallel to the periodic crystals, the study of light propagation in 1D and 2D quasiperiodic photonic crystals has also aroused significant interest [7-10]. Kahn et al. [11] studied the nonlinear property of the 1D Fibonacci quasiperiodic superlattice, and they found no evidence of gap solitons. To our knowledge, the study of the nonlinear property of the 2D quasicrystals is still lacking. An important question is whether stable regular gap solitons can exist in nonlinear 2D quasicrystals, so as to achieve a dynamical tunability of their optical properties as in 2D periodic crystals.

In this paper, different from the previous studies of infinite $2 \mathrm{D}$ crystals $[4,5]$, we demonstrate the existence of gap solitons in a finite-sized 2D nonlinear periodic crystal by studying the multistability of the transmitted energy through the crystal, mimicking the experimental situations. In addition to single-soliton states, for the first time to our knowledge, we demonstrate the existence of two-soliton, three- soliton, and four-soliton trains in the 2D periodic crystal. We also first demonstrate the existence of the symmetric, regular gap soliton, asymmetric single-soliton state, and two-soliton state in $2 \mathrm{D}$ quasiperiodic crystals. We reveal that the existence of the symmetric, regular soliton in the $2 \mathrm{D}$ quasicrystal is determined by the geometrical size of the unit hexagon that forms the core of the dodecahedral cell, which is the building block of 12-fold quasicrystals [9].

Periodic crystal. We consider the square periodic crystal depicted in the inset of Fig. 1(b). The crystal is made of 11 $\times 11+11 \times 10=231$ infinitely long parallel cylinders, each possessing Kerr nonlinearity. The period of the lattice is taken as $a$, and the radius of the cylinders is $R=0.1 a$. An $s$-polarized external beam, in the form of either a slit or a plane wave at a frequency $\omega=2 \pi f$, is incident upon the crystal from the left face. The electric field obeys the Helmholtz equation

$$
\nabla^{2} E(x, y)+\frac{\omega^{2}}{c^{2}} \varepsilon(x, y) E(x, y)=0,
$$

where $\varepsilon(x, y)=\varepsilon_{s}$ in the background medium and

$$
\varepsilon(x, y)=\varepsilon_{0}+\lambda|E(x, y)|^{2}
$$

in the Kerr nonlinear cylinders. Here $\varepsilon_{0}$ is the linear dielectric constant and $\lambda$ is the Kerr coefficient of the cylinders. In this paper we will use the method of multiple scattering [12-14] combined with an iterative scheme to solve Eq. (1). The multiple-scattering method is numerically exact. In order to calculate $\varepsilon(x, y)$ we first consider the intensity distribution $|E(x, y)|^{2}$ inside the photonic crystal for the linear case, i.e., $\lambda=0$. We have checked the intensity distribution when the crystal is illuminated by an external beam with the frequency inside the pass band, at the band-edge state, and inside the band gap, but near the band edge. We noted that, although the mean intensity in different cylinders can have large variations, the intensity distribution in each cylinder is, to a very good approximation, always cylindrically symmetrical. From Eq. (2) we thus deduce that, as we will show later, the intensity distribution $|E(x, y)|^{2}$ in every cylinder for $\lambda \neq 0$ is also, to a very good approximation, cylindrically 


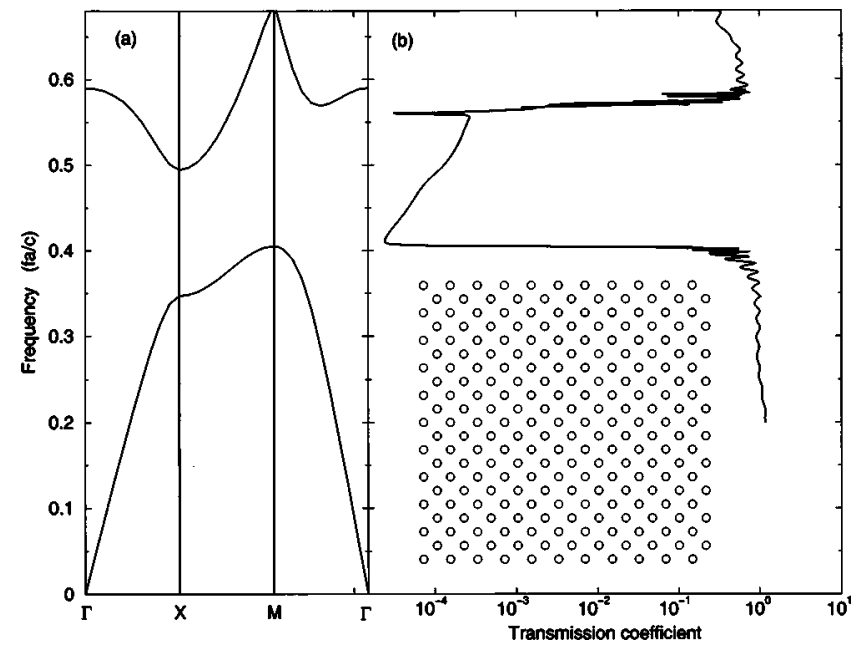

FIG. 1. (a) Band structure of the $s$ wave for the square lattice with $R / a=0.1$ and $\varepsilon_{0}=13$. (b) Transmission coefficient for the linear crystal depicted in the inset.

symmetrical. Therefore we have a very good approximate way to calculate the distribution of $\varepsilon(x, y)$ in the cylinder: In performing the multiple-scattering calculation we divide each cylinder into many cylindrical layers and assume the electric energy in each layer to be constant and equal to the mean intensity $\left\langle|E(x, y)|^{2}\right\rangle$ in that layer. The iterative scheme is briefly as follows: From the value $E_{n}(x, y)$ of the $n$th step we can obtain $\varepsilon_{n+1}(x, y)=\varepsilon_{0}+\lambda\left|E_{n}(x, y)\right|^{2}$. With this new $\varepsilon_{n+1}(x, y)$ we can obtain $E_{n+1}(x, y)$ of the ( $n$ $+1)$ th step from the multiple-scattering method. The procedure is repeated until the relative value $\mid \varepsilon_{n+1}(x, y)$ $-\varepsilon_{n}(x, y) \mid / \varepsilon_{n}(x, y)$ is smaller than a required value $\delta$ (in this paper we take $\left.\delta=10^{-4}\right)$. For the sake of convergence, it is necessary to use $\tilde{\varepsilon}_{n+1}=\varepsilon_{n+1}+\left(\varepsilon_{n+1}-\varepsilon_{n}\right) r$ to replace $\varepsilon_{n+1}$, where $r<1$ is a constant that should be chosen appropriately in the calculation. Throughout this paper we take

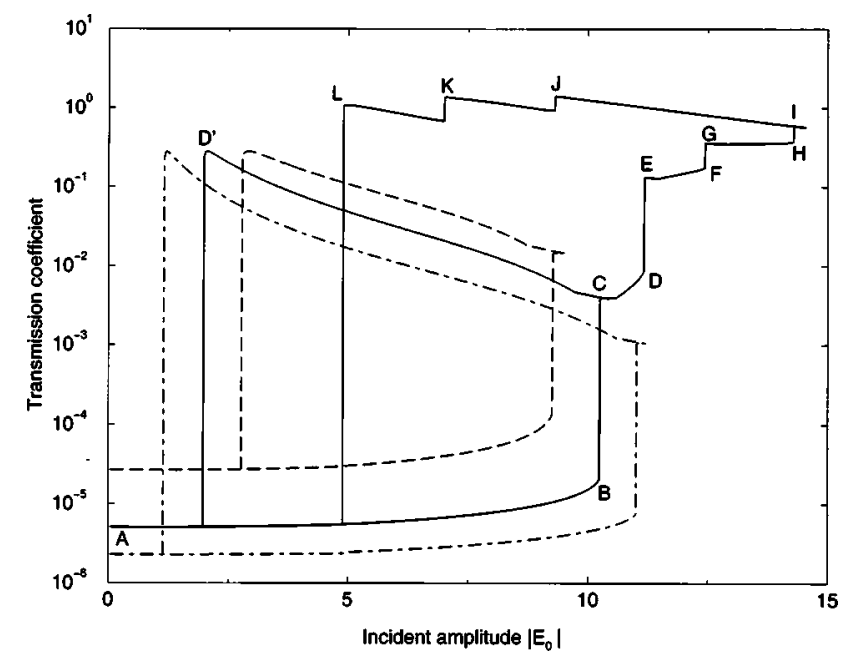

FIG. 2. Transmission coefficient as a function of the incident amplitude in the nonlinear periodic $2 \mathrm{D}$ crystal for $d / 2=1.414 a$ and the dimensionless frequency $\widetilde{f} \equiv f a / c=0.406$ (dashed curve), $\widetilde{f}$ $=0.407$ (solid curve), and $\tilde{f}=0.408$ (dot-dashed curve).

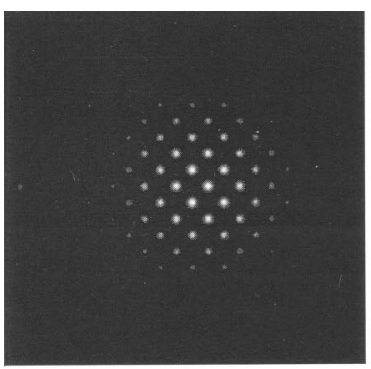

(a)

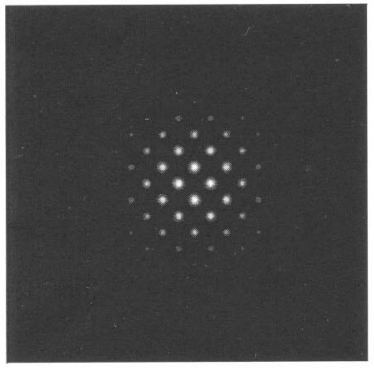

(b)

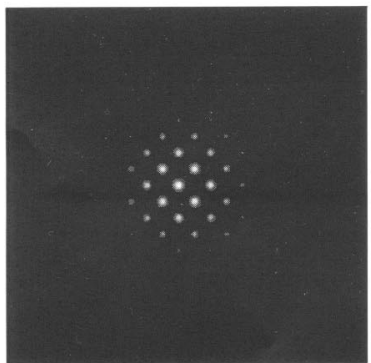

(c)

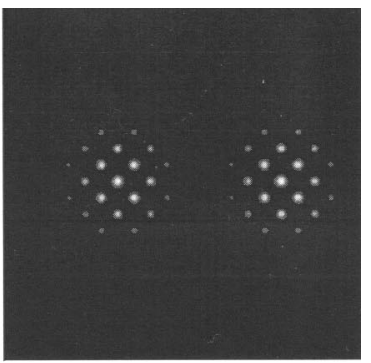

(d)

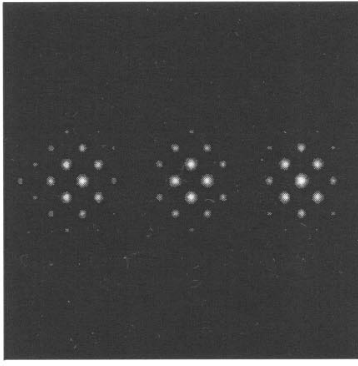

(e)

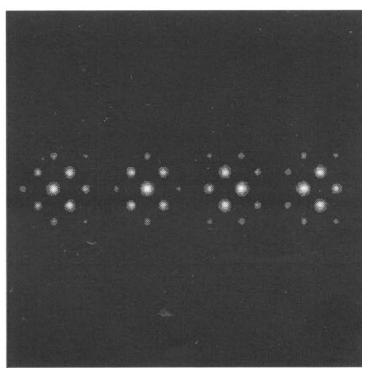

(f)
FIG. 3. Intensity distributions of the solitons in the nonlinear periodic $2 \mathrm{D}$ crystal described in the text. (a) $\tilde{f} \equiv f a / c=0.406$, (b) and (d)-(f) $\tilde{f}=0.407$, and (c) $\tilde{f}=0.408$.

$\varepsilon_{s}=1$ and $\varepsilon_{0}=13$. As to the magnitude of $\lambda$, we pick the one that is normally used in the literature, i.e., $\lambda=-0.001$ $[2,3,14]$.

Figures 1(a) and 1(b) show, respectively, the calculated band structure and the transmission coefficient (defined in Ref. [12]) for the case of $\lambda=0$. There exists a full gap, which extends from $\widetilde{f} \equiv f a / c=0.405$ to $\widetilde{f}=0.494$, where $\widetilde{f}$ is the dimensionless frequency. The transmission coefficient shows excellent agreement with the partial gap, which extends from $\widetilde{f}=0.405$ to $\tilde{f}=0.569$ along the $\Gamma-M$ direction.

To observe the gap soliton, we calculate the transmission coefficient as a function of the amplitude $\left|E_{0}\right|$ of the incident field for a fixed frequency inside the gap near the lower band edge, as shown in Fig. 2. For instance, for $\widetilde{f}=0.407$ (solid curve) we observe a hysteresis loop. As the incident amplitude increases from $A$ to $B$, the beam is in the highly reflecting state. At $B$ it jumps to the transmitting state $C$. Then from $C$, if we decrease the incident amplitude, a maximum of the transmission coefficient occurs at $D^{\prime}$. Figure 3(b) shows the intensity distribution at maximum $D^{\prime}$, which corresponds to a single-soliton state. To compare it with other solitons of different frequencies, in Figs. 3(a) and 3(c) we show, respec- 


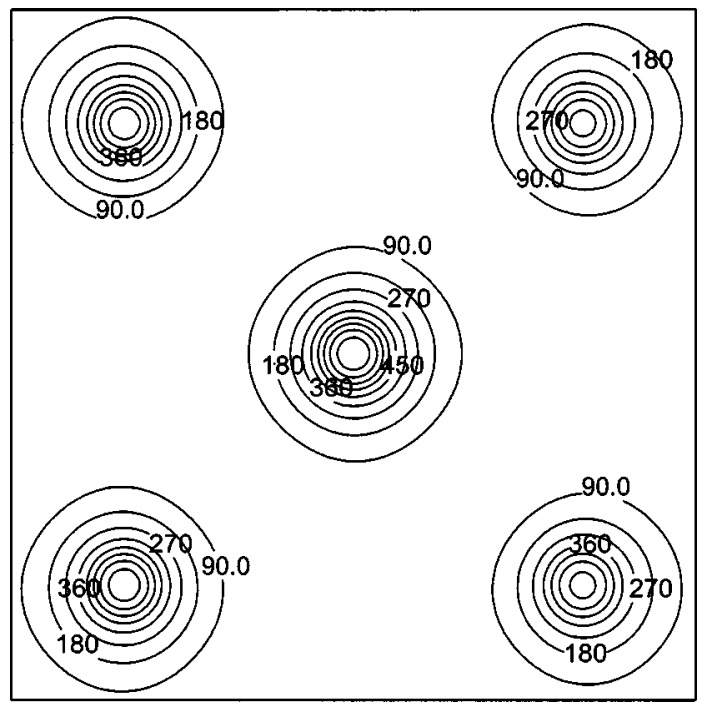

FIG. 4. Contour plots of the intensity profile inside the five cylinders near the center of Fig. 3(b).

tively, solitons for $\tilde{f}=0.406$ (dashed curve) and $\tilde{f}=0.408$ (dot-dashed curve). As is well known, we see that the spatial size of the solitons shrinks as the frequency is moved away from the gap edge. From Figs. 3(a)-3(c) we note that the intensity distribution in each cylinder is cylindrically symmetrical, although the intensity varies greatly at different cylinders. For clarity, in Fig. 4 we show the contour plots of the intensity profile inside the five cylinders near the center of Fig. 3(b). We have also checked that at any other state along $A$ to $B$ and along $C$ to $D^{\prime}$ the intensity distribution is cylindrically symmetrical in each cylinder. This justifies our method of calculating $\varepsilon(x, y)$ that the intensity in each cylindrically layer is constant and can be taken to be the mean intensity in that layer. Here it is important to point out that the solitons shown in Figs. 3(a)-3(c) and the ensuing Figs. 3(d)-3(f) are intrinsic excitations of the system. They do not depend on the external source. We have checked this claim by changing the width of the source slit from $0.5 a$ to the plane-wave source. It has been found that the shapes and energies of these solitons remain unchanged. The only difference is that the jumping transmission $B$ and the maximum $D^{\prime}$ of the transmission coefficient occur at different incident amplitudes $\left|E_{0}\right|$ for different source widths.

Again, we come back to the solid curve in Fig. 2 for $\tilde{f}$ $=0.407$. At point $C$, if we do not decrease, but continue to increase the incident amplitude, we find that the transmission coefficient jumps from $D$ to $E$. A further increase in the incident amplitude leads to another jump at $F$. If we further increase the incident amplitude, another jump from $H$ to $I$ occurs. Then from $I$, if we decrease the incident amplitude, there occurs a maximum of the transmission coefficient at $J$. A further decrease in the incident amplitude leads to another maximum $K$ of the transmission coefficient and another maximum $L$. It should be mentioned that if we decrease the incident amplitude from $G$, we will directly arrive at the maximum $K$, and if we decrease the incident amplitude from $E$, we directly reach maximum $L$. Thus we see that, in some

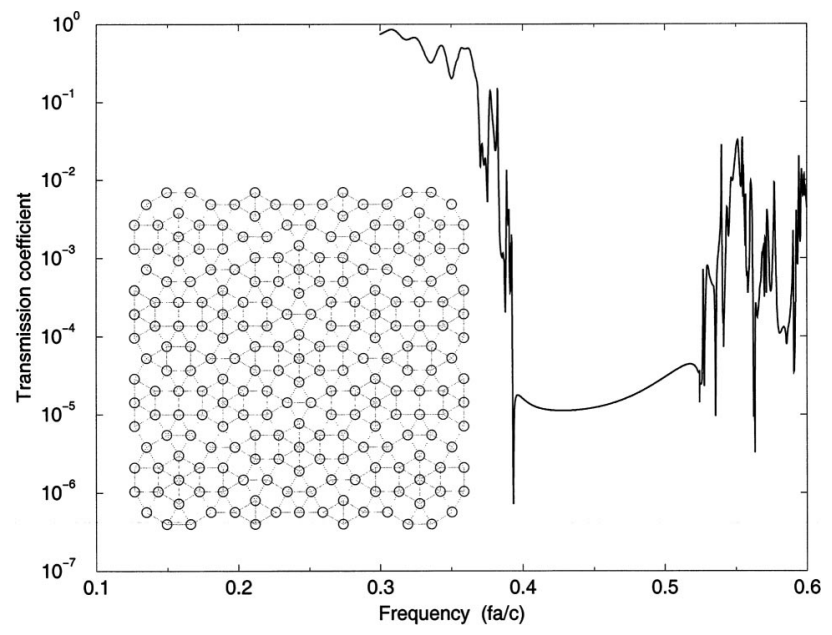

FIG. 5. Transmission coefficient for the linear quasicrystal depicted in the inset.

region of the incident amplitude, multistability of the transmission coefficient occurs. The intensity distributions in the crystal for maxima $L, K$, and $J$ are shown in Figs. 3(d), 3(e), and $3(\mathrm{f})$, respectively. We see that they correspond to two solitons, three solitons, and four solitons, which are called soliton trains [6]. It is evident that if we continue to increase the incident amplitude from point $I$, we should observe a five-soliton train and six-soliton train, etc., if the sample is sufficiently large.

Quasiperiodic crystal. We use the 12 -fold symmetric quasiperiodic crystal depicted in the inset of Fig. $5[9,10]$. The distance between two cylinders is taken to be $a$, and the radius of the cylinders is $0.1 a$. The quasicrystal is made of 225 infinitely long parallel cylinders, with the size of the crystal being $7.3 a \times 7.3 a$. The linear dielectric constant $\varepsilon_{0}$ and the Kerr coefficient $\lambda$ of the cylinder material are the same as those in the periodic case. An $s$-polarized beam from a slit source or a plane-wave source at a frequency $\omega$ $=2 \pi f$ is incident upon the crystal from the left face. The transmission coefficient for the linear quasicrystal is shown in Fig. 5. There exists a gap, which extends approximately from $\widetilde{f}=0.393$ to $\widetilde{f}=0.525$. This is a full gap, which is verified by the calculated radiation power in all direction from a line source positioned near the center of the crystal [10].

Using a similar procedure to that used in the periodic case, we obtain a gap soliton for $\widetilde{f}=0.3945$ [Fig. 6(a)] and that for $\widetilde{f}=0.396$ [Fig. 6(b)] when all cylinders are Kerr nonlinear. It is interesting to see that a gap soliton with symmetric, regular envelopes occurs in the 2D quasicrystal. The electric energy prefers to accumulate in the six cylinders that form a hexagon located at the center of the sample, as shown in the inset of Fig. 5, whereas the energy in the center cylinder within the six hexagonal cylinders is very small. It should be pointed out that the hexagon is the core of the dodecahedral cell, which is the building block of the 12-fold quasicrystals. The energy distribution of the soliton shown in Figs. 6(a) or 6(b) is different from that in the 2D periodic crystal where the energy in every cylinder inside the localized region of the soliton is very high (see Fig. 3). It should 


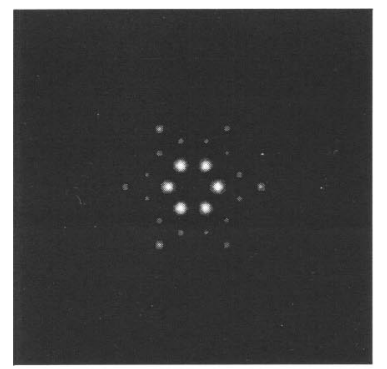

(a)

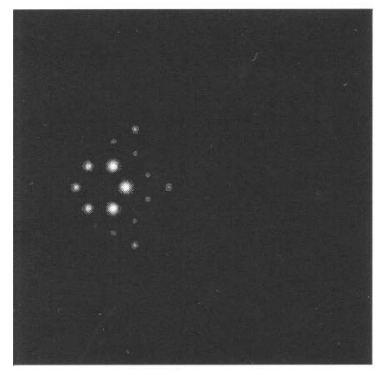

(c)

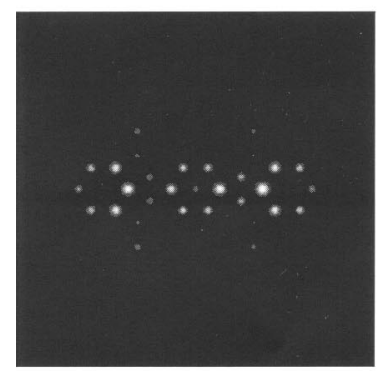

(e)

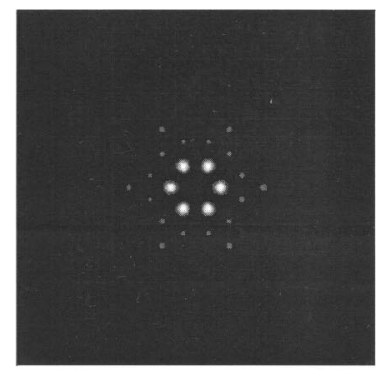

(b)

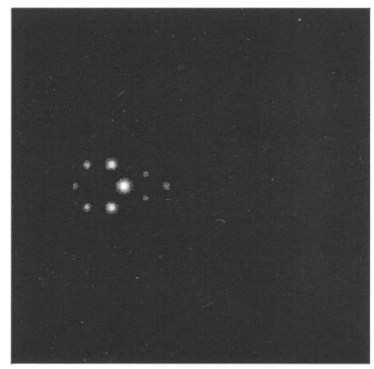

(d)

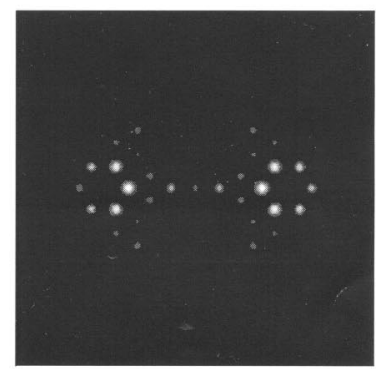

(f)
FIG. 6. Intensity distributions of the solitons in 12-fold nonlinear quasicrystal described in the text. (a) $\widetilde{f} \equiv f a / c=0.3945$, (b) $\widetilde{f}$ $=0.396$, (c) $\tilde{f}=0.396$, (d) $\tilde{f}=0.4$, (e) $\tilde{f}=0.396$, and (f) $\tilde{f}=0.397$.

be mentioned that symmetric gap solitions do not exist in 1D quasiperiodic superlattices [11].

Besides the existence of the type of the symmetric, regular gap solitons given in Figs. 6(a) and 6(b), we also find other types of gap solitons in 2D quasicrystals, as shown in Figs. 6(c)-6(f). It is seen that the electric energy again prefers to accumulate in the six cylinders that form the unit hexagon located at other positions of the sample. The asymmetric solitons shown in Figs. 6(c) and 6(d) are particularly interesting. They originate from the nontranslational invariance of the quasicrystal and are intrinsic excitations of the system. In a periodic photonic crystal, asymmetric intensity profiles can also be excited in a finite-sized sample whenever the transmission is not at the local maximum of a hysteresis loop. For example, in Fig. 2, only the excitations at points $D^{\prime}, L, K$, and $J$ produce intrinsic solitons with symmetric intensity profiles. All other excitations at other points on the hysteresis produce asymmetric intensity profiles. Unlike solitons, the shapes and energies of these excitations depend on the intensity profile of the incident wave. In one-dimensional periodic systems, asymmetric intensity profiles exist whenever the transmission is not unity. They do not represent intrinsic soliton solutions. Solitions appear only when the transmission becomes unity. At these excitations, the intensity profiles are always symmetric. In order to confirm that the asymmetric solitons shown in Figs. 6(c) and 6(d) are intrinsic states of the quasicrystal, not because of the asymmetry of the incident beam relative to the quasicrystal, we have used two external beams of the same frequency and same beam width incident upon the crystal from the left and right faces simultaneously. Although the system now becomes symmetrical, we have obtained the same asymmetric solutions as in Fig. 6(c) and 6(d), including the same symmetric solutions as in Figs. 5(a), 5(b), 5(e), and 5(f). It should be emphasized that, similar to the periodic case, all solitons can be excited by use of the slit source of any width or plane-wave source, with the only difference being that a soliton occurs at different incident intensities for different widths. However, their shapes and energies are independent of the width. It is interesting to mention that, for the same frequency and beam width, the asymmetric type of solitons in Fig. 6(c) or 6(d) occurs at larger incident intensity than that of the symmetric solitons in Fig. 6(a) or 6(b), and the type of soliton trains in Fig. 6(e) or 6(f) occurs at larger incident intensity than that of the single solitons in Fig. 6(c) or $6(\mathrm{~d})$. For example, for frequency $\widetilde{f}=0.396$ and half slit width $d / 2=5.2 a$, the solitons in Figs. 6(b), 6(c), and 6(e) occur at $\left|E_{0}\right|=0.366,1.142$, and 1.81 , respectively. Thus, in contrast to the periodic case where only one type of the single-soliton state exists for a fixed frequency, in the 12-fold quasiperiodic case, there exist two types of the single-soliton state for our sample. One is central symmetrical [Fig. 6(b)], and the other one is asymmetrical [Fig. 6(c)]. The difference is due to the absence of the translation symmetry in quasiperiodic crystal. It should be mentioned that solitons in Figs. 6(e) and 6(f) belong to the same type of the solitons, which corresponds to the two-soliton states in the periodic case. As the frequency is moved into the gap center, the two solitons become apart.

Similar to the periodic case, Fig. 6 shows that, as the frequency is moved away from the gap edge, the spatial size of the solitons shrinks. Here we reveal that, in sharp contrast to the $2 \mathrm{D}$ periodic photonic crystal discussed above, the existence condition of the type of the gap solitons shown in Figs. 6(a) and 6(b) is determined by the geometrical size of the hexagon at the center of the quasicrystal. In other words, as the frequency is moved into the gap, the spatial size of this type of the soliton is reduced, and when the size of the soliton becomes smaller than the geometrical size of the hexagon, the soliton cannot exist. This assertion is consolidated by our careful numerical analysis. In our sample, the soliton becomes nonexistent when $\widetilde{f}$ is larger than $\sim 0.3962$. However, for the gap solitons in the periodic case shown in Fig. 3 and those in the quasiperiodic case shown in Figs. 6(c)-6(f), the geometry of the crystal has no constraint on the existence of the soliton. For the frequency far away from the gap edge, the existence of the stable gap solitons is determined by their stability requirements (see, e.g., Ref. [5]). In our sample, we see that for $\widetilde{f}=0.4$ the stable soliton of type shown in Fig. 6(d) still exists. 
In conclusion, by using the multiple-scattering approach in conjunction with an iterative scheme, we demonstrated the existence of stable gap solitons and soliton trains in finitesized 2D nonlinear periodic photonic crystals. In 12-fold symmetric nonlinear photonic quasicrystals, we also found stable symmetric, regular gap solitons, which is in contrast to 1D Fibonacci quasiperiodic superlattices. In addition, we found asymmetric single-soliton states and two-soliton states which are due to the absence of the translation invariance of the quasiperiodicity. It is revealed that the existence condition of the symmetric, regular gap solitons in a $2 \mathrm{D}$ quasicrystal is determined by its geometrical size of the unit hexagon that forms the core of dodecahedral, which is the building block of 12-fold symmetric quasicrystals.

This work was supported by Hong Kong Research Grants Council Grant No. HKUST 6163/01P.
[1] See, for example, Photonic Band Gap Materials, edited by C. M. Soukoulis (Kluwer Academic, Dordrecht, 1996); J. D. Joannopoulos, R. D. Meade, and J. N. Winn, Photonic Crystal-Molding the Flow of Light (Princeton University Press, Princeton, NJ, 1995); E. Yablonovitch, Phys. Rev. Lett. 58, 2059 (1987); S. John, ibid. 58, 2486 (1987); K. Sakoda, Optical Properties of Photonic Crystals (Springer-Verlag, Berlin, 2001).

[2] M. Scalora et al., Phys. Rev. Lett. 73, 1368 (1994).

[3] W. Chen and D. L. Mills, Phys. Rev. Lett. 58, 160 (1987).

[4] S. John and N. Akozbek, Phys. Rev. Lett. 71, 1168 (1993); N. Akozbek and S. John, Phys. Rev. E 57, 2287 (1998).

[5] S. F. Mingaleev and Y. S. Kivshar, Phys. Rev. Lett. 86, 5474 (2001).

[6] L. Kahn, N. S. Almeida, and D. L. Mills, Phys. Rev. B 37,
8072 (1988).

[7] M. Kohmoto et al., Phys. Rev. Lett. 58, 2436 (1987); R. Merlin et al., ibid. 55, 1768 (1985); M. Nakayama et al., Phys. Rev. B 36, 3472 (1987).

[8] Y. S. Chan, C. T. Chan, and Z. Y. Liu, Phys. Rev. Lett. 80, 956 (1998).

[9] M. E. Zoorobet et al., Nature (London) 404, 740 (2000).

[10] X. Zhang, Z. Q. Zhang, and C. T. Chan, Phys. Rev. B 63, 081105 (2001).

[11] L. M. Kahn, K. Huang, and D. L. Mills, Phys. Rev. B 39, 12449 (1989).

[12] L. M. Li and Z. Q. Zhang, Phys. Rev. B 58, 9587 (1998).

[13] G. Tayeb and D. Maystre, J. Opt. Soc. Am. A 14, 3323 (1997).

[14] E. Centeno and D. Felbacq, Phys. Rev. B 62, R7683 (2000). 